\title{
Zariski chambers, volumes, and stable base loci
}

\author{
Th. Bauer, A. Küronya, T. Szemberg
}

Version of October 30, 2018

\section{Introduction}

In this paper we consider certain asymptotic invariants of linear systems on algebraic surfaces. Originally we were interested in understanding how the volume of a line bundle and its stable base locus behaves with respect to (small) perturbations of the considered bundle. These are asymptotic variants of questions studied classically in algebraic geometry. The asymptotic approach emerged only recently and quickly gained considerable interest.

Let $X$ be a smooth projective variety of dimension $n, L$ a line bundle on $X$. The Riemann-Roch problem is concerned with the study of the behaviour of $h^{0}(X, k L)$ as a function of $k$. The exact determination of $h^{0}$ of a line bundle is difficult in general. The groups in question typically grow like $k^{\operatorname{dim} X}$ so we can introduce their asymptotic counterpart, the notion of the volume of a line bundle $L$

$$
\operatorname{vol}_{X}(L) \stackrel{\text { def }}{=} \limsup _{k} \frac{h^{0}\left(X, L^{\otimes k}\right)}{k^{n} / n !},
$$

which behaves much more nicely in many important cases. The volume was first introduced by Cutkosky (notably in his proof of non-existence of Zariski decomposition for divisors on higher dimensional varieties) and subsequently studied by Demailly, Ein, Lazarsfeld and others.

The concept readily extends — via homogeneity - to $\mathbb{Q}$-divisors and quite generally, it enjoys many useful properties. It has been established recently (in [11) that on an irreducible projective variety of dimension $n$ the volume defines a continuous function on the Néron-Severi space. Also, the volume is log-concave and homogeneous of degree $n$.

Still, there have been few instances worked out in the literature so far. Here we show that the volume function on the cone of big divisors on an algebraic surface is piecewise polynomial (more precisely, the big cone splits into subcones on which the volume function is quadratic).

For the base loci of linear series the situation is similar. Whereas determining the base locus of a given line bundle is quite difficult in general, its asymptotic version is somehow easier to study and exhibits better behavior. Given a line bundle $L$, the stable base locus $\mathrm{SB}(L)$ of $L$ is the intersection of the base loci of the linear series $|k L|$ for all positive integers $k$. More generally, we consider the stable base loci of $\mathbb{Q}$-line bundles $L$ by passing to an integral multiple of $L$; this is well-defined, since the stable base locus is invariant 
under taking multiples (i.e. tensor powers) of a line bundle. These objects were recently studied by Nakamaye (13, [14]). He showed in particular that stable base loci of slightly perturbed nef divisors remain constant in a small neighborhood of the perturbed divisor. Here we investigate in more detail the regions (in the big cone) where the stable base loci remain constant. These regions in the case of surfaces turn out to be convex polyhedral subcones.

Both problems thus lead to a partition of the big cone into suitable subcones, and it is natural to ask whether the partitions agree. Somewhat surprisingly, we show that this is indeed the case and that in fact both problems are closely related to the variation of the Zariski decomposition, which is an interesting problem quite on its own.

More precisely, knowing the Zariski decomposition of a $\mathbb{Q}$-divisor provides a quick way to determine both the volume and the stable base locus of the divisor. Therefore the description of the regions where the support of the negative part of Zariski decompositions is constant will settle both questions we originally studied.

Our main result is the following

Theorem. Let $X$ be a smooth projective surface. Then there is a locally finite decomposition of the big cone of $X$ into rational locally polyhedral subcones such that the following holds:

(i) In each subcone the support of the negative part of the Zariski decomposition of the divisors in the subcone is constant.

(ii) On each of the subcones the volume function is given by a single polynomial of degree two.

(iii) In the interior of each of the subcones the stable base loci are constant.

In addition, we work out in detail the volume function on del Pezzo and $K 3$ surfaces and explore the connections with the action of the Weyl group. In contrast to the case of surfaces we establish the following theorem.

Theorem. For every $n \geq 3$ there exists a smooth projective variety of dimension $n$ such that its corresponding volume function is not locally polynomial.

The organization of the paper goes as follows. In Section 1 we consider the problem of variation of Zariski decompositions in the big cone of a smooth projective surface. We establish the part of the Theorem which regards Zariski decompositions. Along the way we prove that in the interior of the big cone the nef cone is actually locally polyhedral which gives a strengthening of a result of Campana and Peternell ([3]) on the geometry of the nef boundary.

Section 2 deals with the applications of the results in Section 1 to the description of stable base loci and destabilizing numbers. We prove that on surfaces all destabilizing numbers - with the possible exception of the largest one — of a big divisor $L$ with respect to an ample divisor $A$ are rational. We give a counterexample to this statement in dimension three.

We then move on to describe the volume function on surfaces in Section 3, with detailed computations in the case of del Pezzo surfaces. In addition, we investigate the 
relation between the volume and the Weyl action on K3 surfaces. Lastly, we provide an example of smooth $n$-folds $(n \geq 3)$ where the volume function is not locally polynomial.

Finally, Section 4 contains a few somewhat technical results that are used in the main text. We include them for the sake of completeness as we were not able to find a refererence for them.

Acknowledgements. Thomas Bauer was supported by the DFG grant BA 1559/4. Tomasz Szemberg was partially supported by the KBN grant 2P03A 022 17. The authors would like to thank Mihnea Popa, Zoltán Szabó and Árpád Tóth for interesting and helpful discussions. Special thanks are due to Igor Dolgachev for sharing with us his insight on $K 3$ surfaces and Lawrence Ein and Mircea Mustata for pointing out mistakes in one of the predecessors of this manuscript (due to the second author). Finally, the authors would like to express their gratitude towards Rob Lazarsfeld, the thesis advisor of the second author, for suggesting the cooperation, his support and many useful comments.

\section{Zariski decompositions}

In this section we will prove the following theorem on the variation of the Zariski decomposition in the big cone.

Theorem 1.1 Let $X$ be a smooth projective surface. Then there is a locally finite decomposition of the big cone of $X$ into rational locally polyhedral subcones such that in each subcone the support of the negative part of the Zariski decomposition of the divisors in the subcone is constant.

We will use the following notation. If $D$ is an $\mathbb{R}$-divisor, we will write

$$
D=P_{D}+N_{D}
$$

for its Zariski decomposition, and we let

$$
\operatorname{Null}(D)=\{C \mid C \text { irreducible curve with } D \cdot C=0\}
$$

and

$$
\operatorname{Neg}(D)=\left\{C \mid C \text { irreducible component of } N_{D}\right\} .
$$

Of course $\operatorname{Neg}(D) \subset \operatorname{Null}\left(P_{D}\right)$.

Proposition 1.2 Let $X$ be a smooth projective surface and $P$ a big and nef $\mathbb{R}$-divisor on $X$. Then there is a neighborhood $U$ of $P$ in $N_{\mathbb{R}}^{1}(X)$ such that for all divisors $D \in U$ one has

$$
\operatorname{Null}(D) \subset \operatorname{Null}(P) .
$$

Proof. As the big cone is open, we may choose big (and effective) $\mathbb{R}$-divisors $D_{1}, \ldots, D_{r}$ such that $P$ lies in the interior of the cone $\sum_{i=1}^{r} \mathbb{R}^{+} D_{i}$. We can have $D_{i} \cdot C<0$ only for finitely many curves $C$. Therefore, after possibly replacing $D_{i}$ with $\eta D_{i}$ for some small $\eta>0$, we can assume that

$$
\left(P+D_{i}\right) \cdot C>0
$$


for all curves $C$ with $P \cdot C>0$. We conclude then from $(*)$ that

$$
\operatorname{Null}\left(\sum_{i=1}^{r} \alpha_{i}\left(P+D_{i}\right)\right) \subset \operatorname{Null}(P)
$$

for any $\alpha_{i}>0$. So the cone

$$
U=\sum_{i=1}^{r} \mathbb{R}^{+}\left(P+D_{i}\right)
$$

is a neighborhood of $P$ with the desired property.

Denote by $\mathcal{I}(X)$ the set of all irreducible curves on $X$ with negative self-intersection. Note that if $D$ is a big divisor, then by the Hodge index theorem we have $\operatorname{Null}(D) \subset \mathcal{I}(X)$. For $C \in \mathcal{I}(X)$ denote by $C^{\geqslant 0}$ the half-space $\left\{D \in N_{\mathbb{R}}^{1}(X) \mid D \cdot C \geqslant 0\right\}$ and by $C^{\perp}$ the hyperplane $\left\{D \in N_{\mathbb{R}}^{1}(X) \mid D \cdot C=0\right\}$.

Corollary 1.3 The intersection of the nef cone and the big cone is locally polyhedral, i.e., for every $\mathbb{R}$-divisor $P \in \operatorname{Nef}(X) \cap \operatorname{Big}(X)$ there exists a neighborhood $U$ and curves $C_{1}, \ldots, C_{k} \in \mathcal{I}(X)$ such that

$$
U \cap \operatorname{Nef}(X)=U \cap\left(C_{1}^{\geqslant 0} \cap \ldots \cap C_{k}^{\geqslant 0}\right)
$$

Proof. Let $U$ be a neighborhood of $P$ as in the proposition. We have

$$
\operatorname{Big}(X) \cap \operatorname{Nef}(X)=\operatorname{Big}(X) \cap \bigcap_{C \in \mathcal{I}(X)} C^{\geqslant 0}
$$

and therefore

$$
U \cap \operatorname{Nef}(X)=U \cap \bigcap_{C \in \mathcal{I}(X)} C^{\geqslant 0}
$$

For every $C \in \mathcal{I}(X)$ we have either $U \subset C^{\geqslant 0}$, in which case we may safely omit $C^{\geqslant 0}$ from the intersection in (*), or else $U \cap C^{\perp} \neq \varnothing$. But by choice of $U$, the second option can only happen for finitely many curves $C$. In fact, $U \cap \operatorname{Nef}(X)=U \cap \bigcap_{C \in \operatorname{Null}(P)} C^{\geqslant 0}$.

Let $P$ be a big and nef $\mathbb{R}$-divisor on $X$. The face of $P$ is given by

$$
\operatorname{Face}(P)=\bigcap_{\substack{C \in \mathcal{I}(X) \\ P \in C^{\perp}}} C^{\perp} \cap \operatorname{Nef}(X)=\bigcap_{C \in \operatorname{Null}(P)} C^{\perp} \cap \operatorname{Nef}(X)
$$

so that

$$
\text { Face }(P)=\operatorname{Null}(P)^{\perp} \cap \operatorname{Nef}(X)
$$

Given a big and nef $\mathbb{R}$-divisor $P$, consider the set

$$
\Sigma_{P}=\{D \in \operatorname{Big}(X) \mid \operatorname{Neg}(D)=\operatorname{Null}(P)\} .
$$


One checks that $\Sigma_{P}$ is a convex cone. It will in general be neither open nor closed. In Example 3.5 the chamber $\Sigma_{Q_{2}}$ contains the wall spanned by $0, L$ and $E_{2}$ but it doesn't contain the ray through $L$ nor the wall spanned by $0, L$ and $L-E_{1}$. On the other hand $\Sigma_{L}$ is open. If $A$ is an ample divisor, then $\Sigma_{A}=\operatorname{Nef}(X)$ is closed. This shows that all possibilities for the boundary points can happen.

Our aim is now to show that the cones $\Sigma_{P}$ provide the decomposition that is claimed in the theorem. We start with the following properties of these cones.

Lemma 1.4 Let $P$ and $P^{\prime}$ be big and nef divisors on $X$.

(i) $\Sigma_{P}=\Sigma_{P^{\prime}}$ if and only if $\operatorname{Face}(P)=\operatorname{Face}\left(P^{\prime}\right)$.

(ii) $\Sigma_{P} \cap \Sigma_{P^{\prime}}=\varnothing$, if $\operatorname{Face}(P) \neq \operatorname{Face}\left(P^{\prime}\right)$.

(iii) $\operatorname{Big}(X)$ is the union of the sets $\Sigma_{P}$.

Proof. (i) and (ii) follow from (11) plus the fact that $\operatorname{Null}(P)^{\perp} \cap \operatorname{Nef}(X)=\operatorname{Null}\left(P^{\prime}\right)^{\perp} \cap$ $\operatorname{Nef}(X)$ implies $\operatorname{Null}(P)=\operatorname{Null}\left(P^{\prime}\right)$.

For (iii), given a big $\mathbb{R}$-divisor $D$, we need to show that there is a big and nef divisor $P$ such that $\operatorname{Neg}(D)=\operatorname{Null}(P)$. Let $\operatorname{Neg}(D)=\left\{C_{1}, \ldots, C_{k}\right\}$, and take any ample divisor $A$. We claim that a divisor $P$ as required can be constructed explicitly in the form $A+\sum_{i=1}^{k} \lambda_{i} C_{i}$ with suitable non-negative rational numbers $\lambda_{i}$. In fact, the conditions to be fulfilled are

$$
\left(A+\sum_{i=1}^{k} \lambda_{i} C_{i}\right) \cdot C_{j}=0 \quad \text { for } j=1, \ldots, k .
$$

This is a system of linear equations with negative definite coefficient matrix $\left(C_{i} \cdot C_{j}\right)$, and Lemma 4.1 guarantees that all components $\lambda_{i}$ of its solution are non-negative. In fact all $\lambda_{i}$ 's must be positive as $A$ is ample.

The following proposition gives a useful characterization of the loci where two or more faces meet.

Proposition 1.5 Let $D$ be a big divisor on $X$. Then $D$ is in the boundary of some $\Sigma_{P}$ if and only if $\operatorname{Neg}(D) \neq \operatorname{Null}\left(P_{D}\right)$.

Proof. Let $D \in \partial \Sigma_{P}$ and let $N_{D}=\sum_{i=1}^{m} a_{i} N_{i}$. We fix a norm $\|\cdot\|$ on $N_{\mathbb{R}}^{1}(X)$. Then for every small $\varepsilon>0$ there exists an element $\alpha \in N_{\mathbb{R}}^{1}(X)$ with $\|\alpha\|<\varepsilon$ such that $\operatorname{Neg}(D+\alpha) \neq \operatorname{Neg}(D)$.

Let $\alpha=\alpha^{\prime}+\alpha^{\prime \prime}$ be the decomposition induced by the direct sum

$$
N_{\mathbb{R}}^{1}(X)=\left\langle P_{D}, N_{1}, \ldots, N_{m}\right\rangle \oplus\left\langle P_{D}, N_{1}, \ldots, N_{m}\right\rangle^{\perp}
$$

and observe that $\operatorname{Neg}\left(D+\alpha^{\prime}\right)=\operatorname{Neg}(D)$ for $\alpha^{\prime}$ of small norm. So we may assume that $\alpha \in\left\langle P_{D}, N_{1}, \ldots, N_{m}\right\rangle^{\perp}$.

Now we take a convex neighborhood $U$ of $P_{D}$ satisfying the condition of Proposition 1.2. Rescaling if necessary we may assume that $P_{D}+\alpha \in U$. Moreover $P_{D}+\alpha$ is not nef as otherwise we would have the Zariski decomposition $D+\alpha=\left(P_{D}+\alpha\right)+N_{D}$. Hence there is a curve $C_{\alpha} \in \mathcal{I} \backslash\left\{N_{1}, \ldots, N_{m}\right\}$ with $\left(P_{D}+\alpha\right) \cdot C_{\alpha}<0$. This implies that for some 
$t \in[0,1]$ we have $\left(P_{D}+t \alpha\right) \cdot C_{\alpha}=0$. So we have $C_{\alpha} \in \operatorname{Null}\left(P_{D}+t \alpha\right)$, which by the choice of $U$ implies $C_{\alpha} \in \operatorname{Null}\left(P_{D}\right)$. This shows the strict inclusion of $\operatorname{Neg}(D)=\left\{N_{1}, \ldots, N_{m}\right\}$ in $\operatorname{Null}\left(P_{D}\right)$.

For the other direction assume that there is an irreducible negative curve $C \in$ $\operatorname{Null}\left(P_{D}\right) \backslash \operatorname{Neg}(D)$. One checks that for $\varepsilon>0$ the divisor $D+\varepsilon C$ has the Zariski decomposition

$$
D+\varepsilon C=P_{D}+\left(N_{D}+\varepsilon C\right) .
$$

Letting $\varepsilon$ converge to 0 this means that $D$ can be approximated by divisors from a different chamber, so it must be in the boundary. (Note that e.g. $D \in \partial \Sigma_{P_{D}}$.)

We give now a description of the interior of the chambers.

Proposition 1.6 The interior of $\Sigma_{P}$ is given by

$$
\left\{D \in \operatorname{Big}(X) \mid \operatorname{Neg}(D)=\operatorname{Null}(P)=\operatorname{Null}\left(P_{D}\right)\right\}
$$

Proof. As the chambers $\Sigma_{P}$ are disjoint, the interior of $\Sigma_{P}$ consists of the points that are not on the boundary of some chamber.

We turn now to the description of the closure of the chambers $\Sigma_{P}$.

Corollary 1.7 Let $P$ be a nef and big divisor. Then

$$
\operatorname{Big}(X) \cap \bar{\Sigma}_{P}=\left\{D \in \operatorname{Big}(X) \mid \operatorname{Neg}(D) \subset \operatorname{Null}(P) \subset \operatorname{Null}\left(P_{D}\right)\right\}
$$

Proof. Let $D$ be in the set on the right. Then $P_{D} \in$ Face $(P)$ because

$$
P_{D} \in \operatorname{Null}\left(P_{D}\right)^{\perp} \cap \operatorname{Nef}(X) \subset \operatorname{Null}(P)^{\perp} \cap \operatorname{Nef}(X)=\operatorname{Face}(P) .
$$

Hence there is a sequence of divisors $Q_{n}$ in the relative interior of $\operatorname{Face}(P)$ converging to $P_{D}$. In particular for every $n$ we have $\operatorname{Null}\left(Q_{n}\right)=\operatorname{Null}(P)$.

On the other hand, as $\operatorname{Neg}(D) \subset \operatorname{Null}(P)$ there exists a sequence $N_{n}$ of negative definite divisors converging to $N_{D}$ such that $\operatorname{Neg}\left(N_{n}\right)=\operatorname{Null}(P)$. (Just add to $N_{D}$ small fractions of curves $C \in \operatorname{Null}(P) \backslash \operatorname{Neg}(D)$ and apply as usual Lemma 4.3.)

Putting things together, we obtain a sequence $D_{n}=Q_{n}+N_{n}$ of divisors converging to $D$. As $D_{n}=Q_{n}+N_{n}$ is by construction the Zariski decomposition, the divisors $D_{n}$ lie all in the interior of $\Sigma_{P}$. This implies that $D \in \bar{\Sigma}_{P}$.

For the other direction, assume that $D \in \operatorname{Big}(X) \cap \bar{\Sigma}_{P}$ and let $D_{n}$ be a sequence of divisors in the interior of $\Sigma_{P}$ converging to $D$. This implies that for every $n$

$$
\operatorname{Neg}\left(D_{n}\right)=\operatorname{Null}(P) \text { and } \operatorname{Null}\left(P_{D_{n}}\right)=\operatorname{Null}(P)
$$

In other words

$$
P_{D_{n}} \in \operatorname{Null}(P)^{\perp} \quad \text { and } \quad N_{D_{n}} \in\langle\operatorname{Null}(P)\rangle
$$

for every $n$. As both spaces are closed and orthogonal to each other, we obtain

$$
P_{D_{n}} \longrightarrow P_{D} \in \operatorname{Null}(P)^{\perp} \quad \text { and } \quad N_{D_{n}} \longrightarrow N_{D} \in\langle\operatorname{Null}(P)\rangle
$$


Dualizing the first condition and using Lemma 4.2 for the second we arrive at

$$
\operatorname{Null}(P) \subset \operatorname{Null}\left(P_{D}\right) \quad \text { and } \quad \operatorname{Neg}(D) \subset \operatorname{Null}(P) .
$$

We give now an explicit description of the chambers $\Sigma_{P}$. Here we use the notation $V^{\geqslant 0}(M)$ for the subcone of $N_{\mathbb{R}}^{1}(X)$ generated by a subset $M \subset N_{\mathbb{R}}^{1}(X)$ and $V^{>0}(M)$ for its interior.

\section{Proposition 1.8}

$$
\operatorname{Big}(X) \cap \bar{\Sigma}_{P}=(\operatorname{Big}(X) \cap \operatorname{Face}(P))+V^{\geqslant 0}(\operatorname{Null}(P))
$$

Proof. We will use the characterization of the closure of $\Sigma_{P}$ we have just obtained. Take a big divisor $D$ for which

$$
\operatorname{Neg}(D) \subset \operatorname{Null}(P) \subset \operatorname{Null}\left(P_{D}\right)
$$

holds. Then $P_{D} \in \operatorname{Null}\left(P_{D}\right)^{\perp} \subset \operatorname{Null}(P)^{\perp}$, hence $P_{D} \in \operatorname{Face}(P) \cap \operatorname{Big}(X)$. On the other hand $\operatorname{Neg}(D) \subset \operatorname{Null}(P)$ implies $N_{D} \in V^{\geqslant 0}(\operatorname{Null}(P))$, so that we have $D \in$ $(\operatorname{Big}(X) \cap \operatorname{Face}(P))+V \geqslant 0(\operatorname{Null}(P))$.

Going in the other direction, pick a big divisor $D \in(\operatorname{Big}(X) \cap \operatorname{Face}(P))+$ $V \geqslant 0(\operatorname{Null}(P))$. This latter is an orthogonal decomposition. Let $Q$ and $M$ be the components of $D$ in its respective parts. Then $Q \cdot M=0, Q$ is nef, and $M$ is a negative definite divisor. Therefore $D=Q+M$ is the Zariski decomposition of $D$ (by the uniqueness of Zariski decompositions). Evidently, $\operatorname{Neg}(D)=\operatorname{Neg}(M) \subset \operatorname{Null}(P)$ (the inclusion coming from Lemma 4.21), and $Q=P_{D} \in \operatorname{Face}(P)$, which implies $\operatorname{Null}(P) \subset \operatorname{Null}\left(P_{D}\right)$.

Proposition 1.9 The interior of the chamber $\Sigma_{P}$ is equal to

$$
\text { rel.int. Face }(P)+V^{>0}(\operatorname{Null}(P)) \text {. }
$$

Proof. Let $D$ be a big divisor in the interior of $\Sigma_{P}$. Then

$$
\operatorname{Neg}(D)=\operatorname{Null}(P)=\operatorname{Null}\left(P_{D}\right),
$$

hence $P_{D} \in$ rel.int. Face $(P)$ and $N_{D} \in V^{>0}(\operatorname{Null}(P))$.

On the other hand, if $D \in$ rel.int. Face $(P)+V^{>0} \operatorname{Null}(P)$ has the decomposition

$$
D=Q+M
$$

with respect to the linear subspaces generated by rel.int. Face $(P)$ and $V^{>0}(\operatorname{Null}(P))$, then $M$ is a negative definite divisor (or zero), $Q \cdot M=0$, and $Q$ is nef, therefore $D=Q+M$ is again the Zariski decomposition of $D$. As $Q \in \operatorname{rel}$.int. Face $(P)$, we have $\operatorname{Null}(P)=$ $\operatorname{Null}(Q)=\operatorname{Null}\left(P_{D}\right)$. Also, $\operatorname{Neg}(D)=\operatorname{Neg}(M)=\operatorname{Null}(P)$. 
It follows in particular that the chambers are locally polyhedral. Within the big cone, the situation is even better:

Proposition 1.10 If Face $(P)$ is contained in $\operatorname{Big}(X)$, then $\Sigma_{P}$ is polyhedral.

Proof. Take a hyperplane $H$ in $N_{\mathbb{R}}^{1}(X)$ cross-secting $\operatorname{Nef}(X)$. Then Face $(P) \cap H$ is compact. Corollary 1.3 implies that every divisor $D \in \operatorname{Face}(P) \cap H$ has an open neighborhood $U$ such that $U \cap \operatorname{Face}(P) \cap H$ is polyhedral. By compactness we conclude that $\operatorname{Face}(P) \cap H$ itself is polyhedral, and this implies that $\operatorname{Face}(P)$ is polyhedral.

On the other hand one can easily have faces that are not polyhedral:

Example 1.11 Take a surface $X$ with infinitely many $(-1)$-curves $C_{1}, C_{2}, \ldots$, and blow it up at a point that is not contained in any of the curves $C_{i}$. On the blow-up consider the exceptional divisor $E$ and the proper transforms $C_{i}^{\prime}$. Since the divisor $E+C_{i}^{\prime}$ is negative definite, we can proceed as in the proof of Lemma 1.4 to construct for every index $i$ a big and nef divisor $P_{i}$ with $\operatorname{Null}\left(P_{i}\right)=\left\{E, C_{i}^{\prime}\right\}$, and also a divisor $P$ such that $\operatorname{Null}(P)=\{E\}$. But then Face $(P)$ meets contains all faces $\operatorname{Face}\left(P_{i}\right)$, and therefore it is not polyhedral.

In order to prove local finiteness of the chamber decomposition we will also make use of the following statement about Zariski decompositions.

Lemma 1.12 If $D$ is a big divisor and $A$ is an ample divisor, then for all $\lambda \geq 0$

$$
\operatorname{Neg}(D+\lambda A) \subset \operatorname{Neg}(D)
$$

Proof. The idea is to proceed by a (finite) induction on the number of elements in $\operatorname{Neg}(D)$.

If $\operatorname{Neg}(D)=\varnothing$, then $D=P_{D}$ is nef, hence $D+A$ is ample and $\operatorname{Neg}(D+A)$ is an empty set as well.

Assume now for $r \geq 1$ that the Lemma holds for all big divisors with at most $r-1$ irreducible components in the negative part of their Zariski decompositions and let $D=$ $P_{D}+N_{D}$ be a big divisor with $N_{D}=\sum_{i=1}^{r} a_{i} N_{i}$. We will prove the following

Claim. There exists a positive number $\varepsilon_{0}>0$ and affine-linear functions $f_{1}, \ldots, f_{r}: \mathbb{R} \rightarrow \mathbb{R}$ such that for $\varepsilon$ with $0 \leqslant \varepsilon \leqslant \varepsilon_{0}$ the Zariski decomposition of $L+\varepsilon A$ is

$$
\left(P+\varepsilon A+\sum_{i=1}^{r}\left(a_{i}-f_{i}(\varepsilon)\right) N_{i}\right)+\sum_{i=1}^{r} f_{i}(\varepsilon) N_{i}
$$

(the expression in brackets being the positive part) and such that $\varepsilon_{0}$ is a zero of one of the functions $f_{i}$. 
We show first that the above claim suffices in order to complete the proof of the Lemma. Indeed, for $\lambda \in\left[0, \varepsilon_{0}\right]$ the statement follows from the claim, whereas for $\lambda \geq \varepsilon_{0}$ we have

$$
D+\lambda A=\left(D+\varepsilon_{0} A\right)+\left(\lambda-\varepsilon_{0}\right) A
$$

and the induction hypothesis applies to $D+\varepsilon_{0} A$.

Turning to the proof of the claim consider the $\mathbb{R}$-divisor

$$
P^{\prime}=P+\varepsilon A+\sum_{i=1}^{r}\left(a_{i}-x_{i}\right) N_{i}
$$

The divisor $L+\varepsilon A$ has the Zariski decomposition

$$
P^{\prime}+\sum_{i=1}^{r} x_{i} N_{i}
$$

if the following conditions are satisfied

$$
\begin{aligned}
& \text { (1) } 0 \leqslant x_{i} \leqslant a_{i} \quad \text { for all } \mathrm{i} \\
& \text { (2) } P^{\prime} \cdot N_{i}=0 \\
& \text { (3) } P^{\prime} \text { is nef }
\end{aligned}
$$

Note that (3) follows from (1) and (2), since $P+\varepsilon A$ is ample. Condition (2) is equivalent to the linear system of equations

$$
S \cdot\left(\begin{array}{c}
x_{1} \\
\vdots \\
x_{r}
\end{array}\right)=\varepsilon\left(\begin{array}{c}
A N_{1} \\
\vdots \\
A N_{r}
\end{array}\right)+\left(\begin{array}{c}
N N_{1} \\
\vdots \\
N N_{r}
\end{array}\right)
$$

where $S$ denotes the intersection matrix of $N$. Since $S$ is negative definite, the system has the unique solution

$$
\left(\begin{array}{c}
x_{1} \\
\vdots \\
x_{r}
\end{array}\right)=\varepsilon S^{-1}\left(\begin{array}{c}
A N_{1} \\
\vdots \\
A N_{r}
\end{array}\right)+\left(\begin{array}{c}
a_{1} \\
\vdots \\
a_{r}
\end{array}\right)
$$

So the $x_{i}$ are linear functions $f_{i}$ of the parameter $\varepsilon$. Furthermore, we find $x_{i} \leqslant a_{i}$, because by Lemma 4.1 below all the entries of the matrix $S^{-1}$ are $\leqslant 0$. Thus the conditions (1) and (2) will be satisfied if we choose $\varepsilon_{o}$ as the smallest zero of the functions $f_{i}$.

Proposition 1.13 The decomposition of the big cone into the chambers $\Sigma_{P}$ is locally finite.

Proof. Denote by $\operatorname{Amp}(X)$ the ample cone of $X$. Every big divisor has an open neighborhood in $\operatorname{Big}(X)$ of the form

$$
D+\operatorname{Amp}(X)
$$

for some big divisor $D$. (In fact, given a big divisor $D_{0}$, there is an ample divisor $A$ such that $D_{0}-A$ is still big, and hence $D_{0} \in\left(D_{0}-A\right)+\operatorname{Amp}(X)$.) Lemma 1.12 implies that only finitely many chambers $\Sigma_{P}$ can meet this neighborhood. 
We give now the proof of the theorem.

Proof of Theorem 1.1. According to Lemma 1.4 the subcones $\Sigma_{P}$ yield a decomposition of the big cone. By definition the support of the negative part of the Zariski decomposition is constant in $\Sigma_{P}$. Prop. 1.8 implies that the $\Sigma_{P}$ are locally polyhedral, and Prop. 1.13 completes the proof.

As a first application, we now show that Zariski decompositions in the big cone are continuos. The proof uses the local finiteness of the chamber structure in an essential way.

Proposition 1.14 Let $\left(D_{n}\right)$ be a sequence of big divisors converging in $N_{\mathbb{R}}^{1}(X)$ to a big divisor $D$. If $D_{n}=P_{n}+N_{n}$ is the Zariski decomposition of $D_{n}$, and if $D=P+N$ is the Zariski decomposition of $D$, then the sequences $\left(P_{n}\right)$ and $\left(N_{n}\right)$ converge to $P$ and $N$ respectively.

Proof. We consider first the case where all $D_{n}$ lie in a fixed chamber $\Sigma_{P}$. In that case we have by definition $\operatorname{Neg}\left(D_{n}\right)=\operatorname{Null}(P)$ for all $n$, so that

$$
N_{n} \in\langle\operatorname{Null}(P)\rangle
$$

and hence $P_{n} \in \operatorname{Null}(P)^{\perp}$. As

$$
N_{\mathbb{R}}^{1}(X)=\operatorname{Null}(P)^{\perp} \oplus\langle\operatorname{Null}(P)\rangle
$$

we find that both sequences $\left(P_{n}\right)$ and $\left(N_{n}\right)$ are convergent. The limit class $\lim P_{n}$ is certainly nef. Let $E_{1}, \ldots, E_{m}$ be the curves in $\operatorname{Null}(P)$. Then every $N_{n}$ is of the form $\sum_{i=1}^{m} a_{i}^{(n)} E_{i}$ with $a_{i}^{(n)}>0$. Since the $E_{i}$ are numerically independent, it follows that $\lim N_{n}$ is of the form $\sum_{i=1}^{m} a_{i} E_{i}$ with $a_{i} \geqslant 0$, and hence is either negative definite or zero. Therefore $D=\lim P_{n}+\lim N_{n}$ is actually the Zariski decomposition of $D$, and by uniqueness the claim is proved.

Consider now the general case where the $D_{n}$ might lie in various chambers. Since the decomposition into chambers is locally finite, there is a neighborhood of $D$ meeting only finitely many of them. Thus there are finitely many big and nef divisors $P_{1}, \ldots, P_{\ell}$ such that

$$
D_{n} \in \bigcup_{i=1}^{\ell} \Sigma_{P_{i}}
$$

for all $n$. So we may decompose the sequence $\left(D_{n}\right)$ into finitely many subsequences to which the case above applies.

\section{Base loci}

In this section we study stable base loci as considered recently by Nakamaye [13], 14].

For an integral divisor $D$ denote by $\mathrm{SB}(D)$ the stable base locus of $D$, i.e., the intersection of the base loci of the linear series $|k D|$ for all positive integers $k$. More generally, 
we will consider the stable base loci of $\mathbb{Q}$-divisors $D$ by passing to an integral multiple of $D$; this is well-defined, since the stable base locus is invariant under taking multiples (i.e. tensor powers) of a line bundle.

In [9] Ein et al. introduced a related notion of stabilized base locus $\mathrm{B}_{+}(M)$ defined as

$$
\mathrm{B}_{+}(M)=\mathrm{SB}(M-A)
$$

for (an arbitrary) sufficiently small ample $\mathbb{Q}$-divisor $A$. This notion has the advantage of being independent of the numerical equivalence class of a divisor, so it can be safely studied in the space $N_{\mathbb{R}}^{1}(X)$. Moreover, since the definition depends on small perturbations of $D$, it extends in a natural manner to $\mathbb{R}$-divisors. Clearly, one has always inclusions

$$
\mathrm{B}_{+}(D+A) \subset \mathrm{B}_{+}(D) \subset \mathrm{B}_{+}(D-A) .
$$

An $\mathbb{R}$-divisor $D$ is called stable if equalities hold, i.e.,

$$
\mathrm{B}_{+}(D+A)=\mathrm{B}_{+}(D)=\mathrm{B}_{+}(D-A)
$$

for all sufficiently small ample $\mathbb{R}$-divisors $A$. A $\mathbb{Q}$-divisor $D$ is stable if and only if $\mathrm{SB}(D)=$ $\mathrm{SB}(D \pm A)$ for $A$ ample and sufficiently small. All divisors in $\operatorname{Big}(X)$ which are not stable are called instable. (Our definition, although stated differently, agrees with definition 1.25 of 9 .)

Definition 2.1 If $D$ is a stable $\mathbb{R}$-divisor, then its chamber of stability is defined as the set

$$
\mathrm{SC}(D)=\left\{D^{\prime} \in \operatorname{Big}(X) \mid \mathrm{B}_{+}\left(D^{\prime}\right)=\mathrm{B}_{+}(D)\right\} \subset N_{\mathbb{R}}^{1}(X)
$$

We will show in this section that, somewhat surprisingly, the chambers of stability agree essentially with the Zariski chambers of Theorem 1.1.

Theorem 2.2 Let $D$ be a stable big $\mathbb{R}$-divisor. Then

$$
\operatorname{int} \mathrm{SC}(D)=\operatorname{int} \Sigma_{P_{D}}
$$

As it suffices to prove the Theorem for $\mathbb{Q}$-divisors we assume from now on that $D$ is a $\mathbb{Q}$-divisor and we work with the stable base loci.

Let $C$ be an irreducible curve on $X$ that is contained in $\operatorname{SB}(D)$. We will say that $C$ is a bounded base component of $D$, if there is a constant $p$ such that the coefficient of $C$ in the base divisor of the linear series $|k D|$ is less than $p$ for all integers $k$ such that $k D$ is an integral divisor. We will call $C$ an unbounded base component otherwise. One checks that this notion is invariant under taking multiples of $D$. By work of Cutkosky and Srinivas [6] one knows that a bounded base component in fact appears with periodic coefficients in the base divisors of the linear series $|k D|$ for large $k$.

We show:

Proposition 2.3 Let $D$ be a big $\mathbb{Q}$-divisor and $A$ an ample $\mathbb{Q}$-divisor on a smooth projective surface $X$. If $C$ is a bounded base component of $D$, then it is an unbounded base component of $D-\varepsilon A$ for all rational numbers $\varepsilon>0$. 
Proof. Let $D=P_{D}+N_{D}$ be the Zariski decomposition of $D$. Then $C$ is a base component of $P_{D}$, since the components of $N_{D}$ are unbounded base components of $D$ according to [15], Theorem 8.1. Since $P_{D}$ is nef and $C$ is a stable base component, we know from [15], Theorem 9.1., that one has $P_{D} \cdot C=0$, so that

$$
\left(P_{D}-\varepsilon A\right) C<0
$$

Therefore $C$ is an unbounded base component of $P_{D}-\varepsilon A$. Note that [15], Corollary 7.2, implies that we have

$$
h^{0}(k(D-\varepsilon A))=h^{0}\left(k\left(P_{D}-\varepsilon A\right)\right)
$$

for all integers $k$ such that the bundles in question are integral, since $\left(P_{D}-\varepsilon A\right) N_{i}<0$ for all components $N_{i}$ of $N_{D}$. So $C$ is an unbounded base component of $D-\varepsilon A$.

The proposition above gives the following corollary.

Corollary 2.4 If D has a bounded base component, then $D$ is instable.

Proof. Let $C$ be a bounded base component of $D$, and let $A$ be an ample divisor. For rational numbers $\beta>0$, Proposition 2.3 implies that $C$ is an unbounded base component of $D-\beta A$. In particular, $C$ is contained in $\operatorname{SB}(D-\beta A)$. Suppose now that $C$ is also contained in $\operatorname{SB}(D+\alpha A)$ for some rational number $\alpha>0$. If $C$ is an unbounded base component of $D+\alpha A$, then it is clearly an unbounded base component of $D$ as well, which contradicts the hypothesis. So $C$ must be a bounded base component of $D+\alpha A$. But then it is an unbounded base component of $D$ by Proposition 2.3, a contradiction again.

The following proposition implies then Theorem 2.2

Proposition 2.5 If $D$ is a stable $\mathbb{Q}$-divisor, then

$$
\operatorname{Neg}(D)=\operatorname{SB}(D)
$$

Proof. For any rational number $\lambda$ one has

$$
\begin{aligned}
& \operatorname{Neg}(D-\lambda A) \\
& \quad \subset \operatorname{SB}(D-\lambda A) \\
& \subset \operatorname{Neg}(L-\lambda A) \cup\{\text { bounded base components of } D-\lambda A\}
\end{aligned}
$$

The last inclusion here follows from the fact that the base components of $P_{D}$ are bounded, because $P_{D}$ is nef and big (see [15, Theorem 10.1]), plus the fact that the stable base locus does not contain any isolated points (see [15, Theorem 6.1]). If $\lambda$ is small enough, then every stable base component must be unbounded by Corollary 2.4 and the claim follows.

Remark 2.6 Note that using the above proposition one can define stable base loci for stable $\mathbb{R}$-divisors. 
Remark 2.7 It is essential to take interiors in the statement of Theorem 2.2 as the boundaries of the chambers may differ. For example, if $A$ is an ample divisor, then $\mathrm{SC}(A)$ is the open ample cone, whereas $\Sigma_{A}$ is its closure - the nef cone.

We conclude this section by showing that in the case of higher dimensional varieties, chambers of stability need not to be rational. To this end it is convenient to introduce the following notion.

Definition 2.8 Let $L$ be a big line bundle and $A$ an ample line bundle on a smooth projective variety $X$. A positive real number $\lambda$ is a destabilizing number of $L$ relative to $A$, if

$$
\mathrm{SB}(L-\alpha A) \subsetneq \mathrm{SB}(L-\beta A)
$$

for all rational numbers $\alpha, \beta$ with $\alpha<\lambda<\beta$.

It is clear that the inclusion $\mathrm{SB}(L-\alpha A) \subset \mathrm{SB}(L-\beta A)$ holds for any rational numbers $\alpha<\beta$. So the destabilizing numbers are by definition those real numbers, where the stable base locus strictly increases, i.e., where one passes from one chamber of stability to the another one. Note that the smallest and the biggest destabilizing number of $L$ relative to $A$ can be conveniently characterized by the conditions of nefness and bigness:

Remark 2.9 (a) If $L$ is ample, then the smallest destabilizing number of $L$ relative to $A$ is the number

$$
\sigma=\sup \{\lambda \mid L-\lambda A \text { is nef }\},
$$

In fact: If $\lambda$ is smaller than $\sigma$, then $L-\lambda A$ is ample, so that $\operatorname{SB}(L-\lambda A)$ is empty; on the other hand, if $\lambda$ is bigger than $\sigma$, then we find a curve in $\operatorname{SB}(L-\lambda A)$ accounting for the fact that the bundle is not nef.

(b) The biggest destabilizing number of $L$ relative to $A$ is the number

$$
\sup \{\lambda \mid L-\lambda A \text { is big }\} .
$$

This is clear from the fact that $\operatorname{SB}(L-\lambda A)$ becomes all of $X$ as $\lambda$ passes this number.

Theorem 2.2 and the rationality statement in Theorem 1.1 imply:

Proposition 2.10 Let $X$ be a smooth projective surface, let $L$ be a big line bundle and $A$ an ample line bundle on $X$. Then all destabilizing numbers of $L$ relative to $A$ with the possible exception of the biggest one are rational numbers.

We will show now that this need not to be the case for higher dimensional varieties. In particular chambers of stability need not to be rational on varieties of dimension $\geq 3$. To this end we revoke an example of Cutkosky [5. Example 1.6] that he used to show the nonexistence of a Zariski decomposition in higher dimensions. The idea simply is that there is an irrational ray in the boundary of the nef cone which is still contained in the big cone. 
Example 2.11 Let $C$ be an elliptic curve with End $C=\mathbb{Z}$ and let $S=C \times C$. Then the nef cone of $S$ is the circular cone

$$
\operatorname{Nef}(S)=\left\{\alpha \in N S(S) \mid\left(\alpha^{2}\right) \geq 0,(\alpha \cdot h) \geq 0\right\}
$$

where $h$ is any ample class. We denote by $\delta$ the diagonal in $S$ and by $f_{1}$ and $f_{2}$ the fibers of the first, respectively second, projection. These divisors generate the Néron-Severi group and their intersection numbers are $\left(f_{1} . f_{2}\right)=\left(f_{1} . \delta\right)=\left(f_{2} . \delta\right)=1$ and $f_{1}^{2}=f_{2}^{2}=\delta^{2}=0$.

Let $f=\delta-f_{1}-f_{2}$ and let $V=\mathbb{P}\left(\mathcal{O}_{S}(f) \oplus \mathcal{O}_{S}\right)$ be the projectivized bundle with the natural projection $\pi: V \longrightarrow S$. We identify $S$ with the zero section of $V$. Let $S_{i}=\pi^{-1}\left(f_{i}\right)$ for $i=1,2$ and let $H$ be an ample divisor on $V$. The divisor $D=H+\alpha S_{1}+\beta S_{2}$ is ample for arbitrary $\alpha, \beta \in \mathbb{Q}_{\geq 0}$ since $S_{1}$ and $S_{2}$ are nef.

It follows that for arbitrary $\gamma \in \mathbb{Q}_{\geq 0}$ the divisor $D(\gamma)=D+\gamma S$ is big and that its nefness need only to be tested on curves contained in $S$. On $S$ we have $d(\gamma)=\left.D(\gamma)\right|_{S}=$ $\left.H\right|_{S}+\alpha f_{1}+\beta f_{2}+\gamma f$.

Since $f$ is not nef, there exists a maximal positive number $\gamma_{0}$ (depending on $\alpha$ and $\beta$ ) such that $d\left(\gamma_{0}\right)$ is nef but not ample. The condition is simply given by $\left(d\left(\gamma_{0}\right)\right)^{2}=0$. For general $\alpha$ and $\beta$ we obtain an irrational value of $\gamma_{0}$ and from now on we fix such a pair of $\alpha$ and $\beta$.

We showed above that on $V$ the divisor $D(\gamma)$ is nef for $0 \leq \gamma \leq \gamma_{0}$ and not nef for $\gamma>\gamma_{0}$. In particular in the plane (in $\left.N^{1}(V)\right)$ spanned by $S$ and $D$ the ray through $D\left(\gamma_{0}\right)$ is irrational.

Now it is easy to find two ample divisors $L$ and $A$ in this plane such that the half-line $L-\lambda A$ meets the given ray at an irrational point.

To be more specific, let $p$ be such an integer that $p \gamma_{0}>1$ and let $L=p D+\left\lfloor p \gamma_{0}\right\rfloor S$ and $A=D$. Then the first destabilizing number is

$$
\lambda_{1}=\frac{p \gamma_{0}-\left\lfloor p \gamma_{0}\right\rfloor}{\gamma_{0}} \notin \mathbb{Q} .
$$

Indeed, $\lambda_{1}$ is the value where the half-line $L-\lambda A$ hits our irrational ray. Since $L-\lambda_{1} A$ is big and $\lambda_{1}$ is irrational it follows from the real valued Nakai-Moishezon criterion [3] that there exists a divisor, in this case obviously $S$, such that $\left(L-\lambda_{1} A\right)^{2} \cdot S=0$. Hence $S \subset \operatorname{SB}(L-\lambda A)$ for $\lambda>\lambda_{1}$ and rational. This shows that $\lambda_{1}$ is a destabilizing number.

\section{Volume of line bundles on surfaces}

The results on the variation of the Zariski decomposition make it possible to describe the behavior of the volume of line bundles on surfaces. Let $X$ be a smooth projective surface. Recall that the volume of a line bundle on $X$ is the nonnegative real number

$$
\operatorname{vol}_{X}(L) \stackrel{\text { def }}{=} \limsup _{k} \frac{h^{0}\left(X, L^{\otimes k}\right)}{k^{2} / 2} .
$$

The definition extends immediately to $\mathbb{Q}$-divisors by the homogeneity of the volume. In the case of surfaces the rays in $N_{\mathbb{R}}^{1}(X)$ determined by elements of $\mathcal{I}$ are all extremal rays 
of the Mori cone of $X([7])$. They also determine the nef cone of $X$ in the sense that it is enough to test the nefness of an effective $\mathbb{Q}$-divisor $D$ by checking the non-negativity of $D^{2}$ and of the intersection numbers of $D$ with the elements of $\mathcal{I}$.

Proposition 3.1 Let $D$ be a big integral divisor, $D=P_{D}+N_{D}$ the Zariski decomposition of $D$. Then

(i) $H^{0}(X, k D)=H^{0}\left(X, k P_{D}\right)$ for all $k \geq 1$ such that $k P_{D}$ is integral, and

(ii) $\operatorname{vol}(D)=\operatorname{vol}\left(P_{D}\right)=\left(P_{D}^{2}\right)$.

By the homogeneity and continuity of the volume we obtain that

Corollary 3.2 For an arbitrary big $\mathbb{R}$-divisor $D$ with Zariski decomposition $D=P_{D}+N_{D}$ we have

$$
\operatorname{vol}(D)=\left(P_{D}^{2}\right)=\left(D-N_{D}\right)^{2} .
$$

Therefore on a chamber $\Sigma_{Q}$ on which the support of the negative part is constant the volume is given by a homogeneous quadratic polynomial.

Corollary 3.3 The volume function $\operatorname{vol}_{X}: N_{\mathbb{R}}^{1}(X) \ni D \longrightarrow \operatorname{vol}(D) \in \mathbb{R}$ of a smooth projective surface is locally polynomial.

\subsection{Del Pezzo surfaces}

We will work out the volume of line bundles on del Pezzo surfaces and describe its connection to the Weyl action on the Picard group. As $-K_{X}$ is ample, del Pezzo surfaces have only a finite number of extremal rays. The corresponding set of hyperplanes (consisting of divisors perpendicular to them) will give a decomposition of the big cone into a finite set of polyhedral chambers on each of which we can write down a polynomial formula for the volume.

Let us establish some notation. We denote by $X=B l_{\Sigma}\left(\mathbb{P}^{2}\right)$ the blow-up of the projective plane at $\Sigma \subseteq \mathbb{P}^{2}$ where $\Sigma$ consists of at most eight points in general position. The exceptional divisors corresponding to the points in $\Sigma$ are denoted by $E_{1}, \ldots, E_{r}(r \leq$ 8). We denote the pullback of the hyperplane class on $\mathbb{P}^{2}$ by $L$. These divisor classes generate the Picard group of $X$ and their intersection numbers are: $L^{2}=1,\left(L . E_{i}\right)=0$ and $\left(E_{i} . E_{j}\right)=-\delta_{i j}$ for $1 \leq i, j \leq r$. For each $1 \leq r \leq 8$ one can describe explicitly all extremal rays on $X$ (see [8]). Recall that a class $\alpha \in \mathrm{NS}(X)$ is a root if $\alpha^{2}=-2$ and $\alpha \cdot K_{X}=0$. We denote the set of roots by $\mathcal{R}$.

Proposition 3.4 With notation as above, the set $\left\{E^{\perp} \mid E \in \mathcal{I}\right\}$ determines the chambers for the volume function. More precisely, we obtain the chambers by dividing the big cone into finitely many parts by the hyperplanes $E^{\perp}$.

Together with the combinatorial description of $\mathcal{I}$ this gives complete information about the volume on $X$. 
Proof. Observe that as the only negative curves on a del Pezzo surface are (-1)-curves, the support of every negative divisor consists of pairwise orthogonal curves. This can be seen as follows. Take a negative divisor $N=\sum_{i=1}^{m} a_{i} N_{i}$. Then, as the self-intersection matrix of $N$ is negative definite, for any $1 \leq i<j \leq m$ one has

$$
0>\left(N_{i}+N_{j}\right)^{2}=N_{i}^{2}+2\left(N_{i} \cdot N_{j}\right)+N_{j}^{2}=2\left(N_{i} \cdot N_{j}\right)-2 .
$$

As $N_{i} \cdot N_{j} \geq 0$, this can only hold if $N_{i} \cdot N_{j}=0$.

According to Proposition 1.5, a big divisor $D$ is in the boundary of a Zariski chamber if and only if

$$
\operatorname{Neg}(D) \neq \operatorname{Null}\left(P_{D}\right) \text {. }
$$

From Lemma 4.3 we see that if $C \in \operatorname{Null}\left(P_{D}\right)-\operatorname{Neg}(D)$ for an irreducible negative curve $C$ then $N_{D}+C$ forms a negative divisor. By the previous reasoning, this implies that $N_{D} \cdot C=0$ hence $D \cdot C=0$, that is, $D \in C^{\perp}$ as required.

Going the other way, if $D \in C^{\perp}$ for an irreducible negative curve $C$ then either $P_{D} \cdot C=0$, that is, $C \in \operatorname{Null}\left(P_{D}\right)$ or $P_{D} \cdot C>0$.

In the first case, $C \notin \operatorname{Neg}(D)$, as otherwise we would have $N_{D} \cdot C<0$ and consequently $D \cdot C<0$ contradicting $D \in C^{\perp}$. Therefore $C \in \operatorname{Null}\left(P_{D}\right)-\operatorname{Neg}(D)$ and $D$ is in the boundary of some Zariski chamber.

In the second case, $D \cdot C=0$ and $P_{D} \cdot C>0$ imply $N_{D} \cdot C<0$. From this we see that $C \in \operatorname{Neg}(D)$ but this would mean $P_{D} \cdot C=0$ which is again a contradiction.

The conclusion is that on a surface on which the only negative curves are $(-1)$-curves, a big divisor $D$ is in the boundary of a Zariski chamber if and only if there exists an (-1)-curve $C$ with $D \in C^{\perp}$.

Example 3.5 (Blow-up of two points in the plane) In this case there are three irreducible negative curves: the two exceptional divisors, $E_{1}, E_{2}$ and the pullback of the line through the two blown-up points, $L-E_{1}-E_{2}$. As we saw in the previous proposition, the corresponding hyperplanes determine the chamber structure on the big cone. They divide the big cone into five regions on each of which the support of the negative part of the Zariski decomposition remains constant.

In this case the chambers are simply described as the set of divisors that intersect negatively the same set of negative curves. In the picture, $A$ is any ample divisor, $P, Q_{1}, Q_{2}$ are big and nef divisors in the nef boundary (hence necessarily non-ample) which are in the relative interiors of the indicated faces. The chambers we obtain in the big cone are $\Sigma_{A}$ (the nef cone itself), $\Sigma_{P}, \Sigma_{Q_{1}}, \Sigma_{Q_{2}}, \Sigma_{P}$ and $\Sigma_{L}$. Observe that apart from the nef cone, the chambers do not contain the nef divisors they are associated to.

Let $D=a L-b_{1} E_{i}-b_{2} E_{2}$ be a big $\mathbb{R}$-divisor. Then one can express the volume of $D$ in terms of the coordinates $a, b_{1}, b_{2}$ as follows:

$$
\operatorname{vol}(D)= \begin{cases}D^{2}=a^{2}-b_{1}^{2}-b_{2}^{2} & \text { if } D \text { is nef, i.e. } D \in \Sigma_{A} \\ a^{2}-b_{2}^{2} & \text { if } D \cdot E_{1}<0 \text { and } D \cdot E_{2} \geq 0 \text { i.e. } D \in \Sigma_{Q_{1}} \\ a^{2}-b_{1}^{2} & \text { if } D \cdot E_{2}<0 \text { and } D \cdot E_{1} \geq 0 \text { i.e. } D \in \Sigma_{Q_{2}} \\ a^{2} & \text { if } D \cdot E_{1}<0 \text { and } D \cdot E_{2}<0 \text { i.e. } D \in \Sigma_{L} \\ 2 a^{2}-2 a b_{1}-2 a b_{2}+2 b_{1} b_{2} & \text { if } D \cdot\left(L-E_{1}-E_{2}\right)<0 \text { i.e. } D \in \Sigma_{P} .\end{cases}
$$




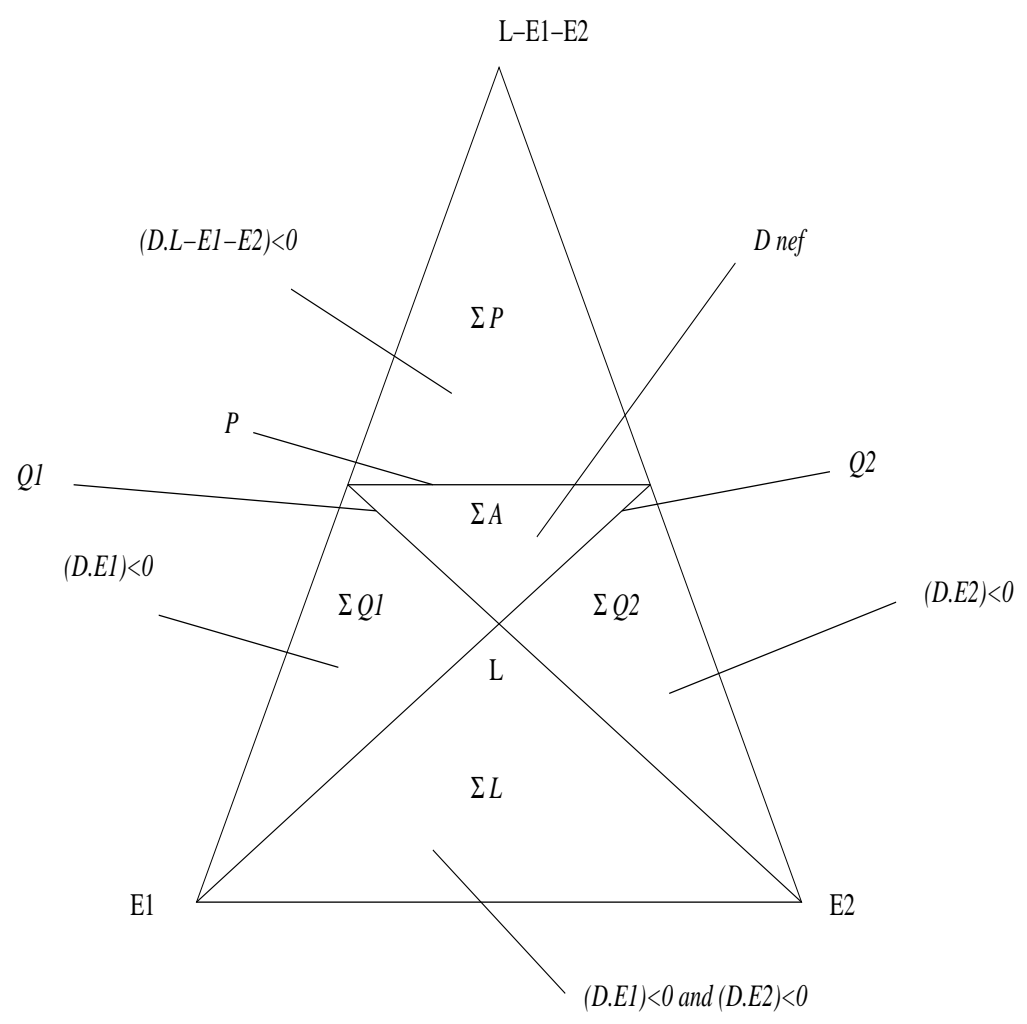

Observe that not all possible combinations of negative divisors occur. This in part is accounted for by the fact that certain faces of the nef cone are not contained in the big cone.

Next we move on to spell out the connection with the Weyl action. To each root $\alpha$ one can associate a reflection of the lattice $\operatorname{Pic}(X)$ :

$$
\sigma_{\alpha}(D)=D+(D \cdot \alpha) \alpha
$$

As a linear automorphism of $\operatorname{Pic}(X)$, every $\sigma_{\alpha}$ descends uniquely to the Néron-Severi space. The group generated by the reflections $\sigma_{\alpha}, \alpha$ a root, is called the Weyl group $W(X)$ of the surface $X$. However, there is a much smaller set of generators. For $r \geq 3$ the roots

$$
\alpha_{1}=L-E_{1}-E_{2}-E_{3}, \alpha_{2}=E_{2}-E_{1}, \ldots, \alpha_{r}=E_{r}-E_{r-1}
$$

called simple roots already generate $W(X)$. The sets of big classes which intersect the same set of roots positively are called Weyl chambers.

Proposition 3.6 Given a del Pezzo surface $X, W(X)$ is the set of automorphisms of $\operatorname{Pic}(X)$ that leave $K_{X}$ fixed. It is finite for $r \leq 8$ and acts transitively on $\mathcal{I}$ (for $r \geq 3$ ) and on $\mathcal{R}$ (for $r \geq 2$ ).

For a proof the reader is referred to [8]. The effect of the Weyl group on the volume and Zariski decompositions is given in the following 
Proposition 3.7 Let $D, D^{\prime}$ be two big divisors that belong to the same Zariski chamber, and let $\sigma \in W(X)$ be an element of the Weyl group. Then:

(i) The supports of the negative parts of $\sigma(D)$ and $\sigma\left(D^{\prime}\right)$ are also equal, ie. $\sigma(D)$ and $\sigma\left(D^{\prime}\right)$ also belong to the same Zariski chamber.

(ii) $\operatorname{vol}(\sigma(D))=\operatorname{vol}(D)$.

Proof. Assume $r \geq 3$, as the remaining cases are easy to check. Then $\sigma$ permutes the exceptional elements hence it takes nef divisors to nef divisors. It is enough to check this statement on the generating reflections so we can assume that $\sigma^{2}=1$. If $C, D$ are any divisors then

$$
(\sigma(D) \cdot C)=(D \cdot \sigma(C))
$$

as $\sigma$ preserves the intersection form. By the previous proposition

$$
\sigma(\mathcal{I})=\mathcal{I}
$$

so $D$ is nef if and only if $\sigma(D)$ is.

Consequently, forming the Zariski decomposition of $D$ commutes with the action of $\sigma$, i.e. if $D=P+\sum_{i=1}^{s} a_{i} N_{i}$ is the Zariski decomposition of $D$ then

$$
\sigma(D)=\sigma(P)+\sum_{i=1}^{s} a_{i} \sigma\left(N_{i}\right)
$$

is the Zariski decomposition of $\sigma(D)$. This proves both statements.

Remark 3.8 We observe that for $r \geq 3$ the Weyl chambers and the volume chambers coincide.

\subsection{K3 surfaces}

The interplay between the volume and the Weyl action on del Pezzo surfaces is in some sense not typical. As we will see, on $K 3$ surfaces, the volume function in not invariant under the action of the Weyl group. However, it is still true that inside the big cone the volume chambers coincide with the Weyl chambers.

Let $Y$ be a projective $K 3$ surface. Then there are no $(-1)$-curves on $Y$ and the Mori cone is generated by either the nef cone itself or by the rational curves with selfintersection 0 and -2 (see [10]). In the first case there is nothing to prove, as every big divisor is ample. In the second case, a big divisor is nef if and only if it intersects every (-2)-curve non-negatively. Hence we can restrict our attention to (-2)-curves.

To every (-2)-curve $E$ one associates as before the reflection

$$
\sigma_{E}(D)=D+(D \cdot E) E
$$

of the Néron-Severi space. These elementary reflections then generate the Weyl group $W(Y)$ of the surface. The Weyl chamber consisting of divisor classes intersecting every 
$(-2)$-curve nonnegatively is called the fundamental Weyl chamber. In our case, it coincides with the nef cone. For terminology and basic results on Weyl groups of $K 3$ surfaces, root systems and related topics the reader should consult [4, 2].

First, we show that contrary to del Pezzo surfaces vol $(D) \neq \operatorname{vol}(\sigma(D))$ in general. Let $P$ be nef divisor on $Y, E$ a $(-2)$-curve such that $(P \cdot E) \neq 0$. The Zariski decomposition of $\sigma_{E}(P)$ is

$$
\sigma_{E}(P)=\left(P+\frac{1}{2}(P \cdot E) E\right)+\frac{1}{2}(P \cdot E) E .
$$

Hence for the volume of $\sigma_{E}(P)$ we obtain

$$
\begin{aligned}
\operatorname{vol}\left(\sigma_{E}(P)\right) & =\operatorname{vol}\left(P+\frac{1}{2}(P \cdot E) E\right) \\
& =\left(\left(P+\frac{1}{2}(P \cdot E) E\right)^{2}\right) \\
& =P^{2}+(P \cdot E)^{2}-\frac{1}{2}(P \cdot E) \\
& =\operatorname{vol}(P)+(P \cdot E)^{2}-\frac{1}{2}(P \cdot E) .
\end{aligned}
$$

As $(P \cdot E) \neq 0$, this is not equal to $\operatorname{vol}(P)$ (one can replace $P$ by a sufficiently high multiple if necessary).

Proposition 3.9 For any $K 3$ surface $Y$, the volume chambers and the Weyl chambers in the big cone are the same.

Proof. Let $D$ be a big divisor on $Y$. By definition $D$ is nef if and only if it belongs to the fundamental Weyl chamber. Therefore we will assume that $D$ is not nef.

Observe that

$$
\Phi_{D} \stackrel{\text { def }}{=}\{E \mid E \text { is a }(-2) \text {-curve with } D \cdot E<0\}
$$

is finite. Also, $\Phi_{D} \subseteq \operatorname{Neg}(D)$ as $D . E<0$ for all $E \in \Phi_{D}$, hence in particular $\left|\Phi_{D}\right|=$ $r \leq \rho=\operatorname{rank} N S(Y)$. Again, by [12, 1.11 we observe that $D=w(P)$, with $P$ nef, $w \in W(Y)$ and $w=\sigma_{E_{1}} \ldots \sigma_{E_{r}}$ a minimal decomposition of $w$ in terms of the reflections corresponding to $(-2)$-curves and $\Phi_{D}=\left\{E_{1}, \ldots, E_{r}\right\}$. But then $\Phi_{D} \supseteq \operatorname{Neg}(D)$ which implies $\Phi_{D}=\operatorname{Neg}(D)$. As Weyl chambers are characterized by $\Phi_{D}$ and volume chambers inside the big cone are characterized by $\operatorname{Neg}(D)$, we can conclude that indeed, for big divisors, every volume chamber is a Weyl chamber and vice versa.

\subsection{An example where the volume is not locally polynomial}

In this section we construct an example of a smooth projective threefold whose associated volume function is not given locally by a polynomial. We use Cutkosky construction as recalled in Example 2.11. We keep the notation introduced in that example. 
We may choose

$$
D=f_{1}+f_{2}, H=3\left(f_{2}+\delta\right)
$$

two ample divisors with classes $d$ and $h$ in such a way that the ray $d-\lambda h$ in $N^{1}(\mathbb{R})$ intersects the boundary of the nef cone at a point $\lambda=\sigma$. Take $A_{1}=D$ and $A_{2}=-H$, and define $\mathcal{E}=\mathcal{O}_{S}\left(A_{1}\right) \oplus \mathcal{O}_{S}\left(A_{2}\right)$ and $X=\mathbb{P}(\mathcal{E})$. Then

$$
h^{0}\left(X, \mathcal{O}_{X}(k)\right)=\sum_{i+j=k} h^{0}\left(S, \mathcal{O}_{S}(i D-j H)\right)
$$

by Lemma 4.4 and

$$
h^{0}\left(S, \mathcal{O}_{S}(i D-j H)\right)=\frac{1}{2}\left((i d-j h)^{2}\right)
$$

if $\frac{j}{i}<\sigma$ and 0 if $\frac{j}{i}>\sigma$ by Riemann-Roch on the abelian surface $S$.

It is important to start with a non-ample line bundle $L=\mathcal{O}_{\mathbb{P}(\mathcal{E})}(1)$ on $X$ as inside the ample cone the volume is a polynomial function given by the self-intersection. Next, we will perturb $L$ by a small $\mathbb{Q}$-divisor depending on a parameter $\varepsilon$ and establish that the dependence of the volume on $\varepsilon$ is not polynomial. Take

$$
A_{1}(\varepsilon)=D+\varepsilon f_{1}, A_{2}(\varepsilon)=-H+\varepsilon f_{1}
$$

then

$$
X=\mathbb{P}\left(\mathcal{O}_{S}\left(A_{1}\right) \oplus \mathcal{O}_{S}\left(A_{2}\right)\right) \simeq \mathbb{P}\left(\mathcal{O}_{S}\left(A_{1}(\varepsilon) \oplus \mathcal{O}_{S}\left(A_{2}(\varepsilon)\right)\right)\right.
$$

for all $\varepsilon$ and

$$
L(\varepsilon) \stackrel{\text { def }}{=} \mathcal{O}_{\mathbb{P}\left(\mathcal{O}_{S}\left(A_{1}(\varepsilon) \oplus \mathcal{O}_{S}\left(A_{2}(\varepsilon)\right)\right)\right.}(1) \simeq L \otimes \pi^{*} \mathcal{O}_{S}\left(\varepsilon f_{1}\right)
$$

Then we have

$$
h^{0}\left(S, \mathcal{O}_{S}\left(i D-j H+(i+j) \varepsilon f_{1}\right)\right)=\frac{1}{2}\left(\left(i \delta-j h+(i+j) \varepsilon f_{1}\right)^{2}\right)
$$

if $\frac{j}{i}<\sigma(\varepsilon)$ and 0 otherwise, where $\sigma(\varepsilon)=\frac{9+5 \varepsilon-\sqrt{45+78 \varepsilon+49 \varepsilon^{2}}}{18-12 \varepsilon}$. Put

$$
q(x)=\left(\left(x d-(1-x) h+\varepsilon f_{1}\right)^{2}\right)
$$

then as in 11] we have

$$
\operatorname{vol}(L(\varepsilon))=3 \int_{\frac{1}{1+\sigma(\varepsilon)}}^{1} q(x) d x .
$$

By plugging in the data of our example we obtain

$$
\begin{aligned}
\operatorname{vol}(L(\varepsilon))= & \frac{1}{\left(-27+7 \varepsilon+\sqrt{45+78 \varepsilon+49 \varepsilon^{2}}\right)^{3}} \times \\
& \times\left(33480 \varepsilon+43128 \varepsilon^{2}+8748-1692 \sqrt{45+78 \varepsilon+49 \varepsilon^{2}}+14120 \varepsilon^{3}\right. \\
& -3300 \varepsilon \sqrt{45+78 \varepsilon+49 \varepsilon^{2}}-2740 \varepsilon^{2} \sqrt{45+78 \varepsilon+49 \varepsilon^{2}} \\
& \left.+84 \varepsilon^{3} \sqrt{45+78 \varepsilon+49 \varepsilon^{2}}+588 \varepsilon^{4}\right)
\end{aligned}
$$


which is not a polynomial function of $\varepsilon$.

By taking a product of $X$ with a projective space of appropriate dimension we can obtain examples in every dimension at least three: for $n \geq 3$ take $Y=X \times \mathbb{P}^{n-3}$, $M_{\varepsilon}=\pi_{1}^{*} L_{\varepsilon} \otimes \pi_{2}^{*} H$, where $H$ is the hyperplane class in $\mathbb{P}^{n}$. Then by the Künneth formula for the volume

$$
\operatorname{vol}_{Y}\left(M_{\varepsilon}\right)=\left(\begin{array}{l}
n \\
3
\end{array}\right) \operatorname{vol}_{X}\left(L_{\varepsilon}\right) \cdot \operatorname{vol}_{\mathbb{P}^{n-3}}(H)=\left(\begin{array}{l}
n \\
3
\end{array}\right) \operatorname{vol}_{X}\left(L_{\varepsilon}\right) .
$$

Therefore the volume function associated to $Y$ is not locally polynomial.

The computations in this section were done with the help of the computer algebra package Maple.

\section{Auxiliary results}

Lemma 4.1 Let $S$ be a negative definite $r \times r$-matrix over the reals such that $s_{i j} \geqslant 0$ for all $i \neq j$. Then all entries of the inverse matrix $S^{-1}$ are $\leqslant 0$.

While we feel that the statement must be well-known, we indicate a proof for lack of a reference.

Proof. We argue by induction on $r$. The case $r=1$ being clear, we assume $r \geqslant 2$. We have

$$
S^{-1}=\frac{1}{\operatorname{det} S} S_{\text {adj }}=\frac{1}{(-1)^{r} \cdot|\operatorname{det} S|} S_{\text {adj }}
$$

so we need to show that all entries of the cofactor matrix $S_{\text {adj }}$ have the $\operatorname{sign}(-1)^{r-1}$. Denoting by $S_{i j}$ the matrix obtained from $S$ by deleting the $i$-th row and the $j$-th column, the assertion is that the numbers $(-1)^{i+j} \operatorname{det} S_{i j}$ have the sign $(-1)^{r-1}$. For $i=j$ this is certainly true, since $S_{i i}$ is negative definite. By symmetry it is then sufficient to consider the case $i<j$. Expanding the determinant $\operatorname{det} S_{i j}$ with respect to the $i$-th column, we get an expression

$$
\operatorname{det} S_{i j}=\sum_{k=1}^{r-1}(-1)^{i+k} \operatorname{det}\left(\left(S_{i j}\right)_{k i}\right) \cdot c_{k}
$$

where $c_{k}$ is a non-negative number. The essential point is now that one has

$$
\operatorname{det}\left(\left(S_{i j}\right)_{k i}\right)=\operatorname{det}\left(\left(S_{i i}\right)_{k, j-1}\right)
$$

The claim follows upon using this relation in equation $(*)$ above and applying the induction hypothesis.

Lemma 4.2 Let $N$ be a negative definite divisor with irreducible components $E_{1}, \ldots E_{m}$. Then the only irreducible curves in the linear span $\left\langle E_{1}, \ldots, E_{m}\right\rangle$ in $N_{\mathbb{R}}^{1}(X)$ are $E_{1}, \ldots, E_{m}$. 
Proof. Let $C$ be any irreducible curve in $\left\langle E_{1}, \ldots, E_{m}\right\rangle$. After re-indexing we can write

$$
C=\sum_{i=1}^{k} \alpha_{i} E_{i}-\sum_{i=k+1}^{m} \beta_{i} E_{i}
$$

where $\alpha_{i}>0$ and $\beta_{i} \geqslant 0$. We must have $k \geqslant 1$, hence

$$
0>\left(\sum_{i=1}^{k} \alpha_{i} E_{i}\right)^{2}=\left(\sum_{i=1}^{k} \alpha_{i} E_{i}\right) \cdot\left(C+\sum_{i=k+1}^{m} \beta_{i} E_{i}\right),
$$

and we conclude that $C$ is one of the curves $E_{i}$.

Lemma 4.3 Let $P$ be a nef and big divisor. Then any non-zero combination of curves in $\operatorname{Null}(P)$ is a negative divisor i.e. a divisor with negative definite intersection form.

Proof. As $P^{2}>0$, this follows directly from the Hodge Index Theorem.

Now we recall, following closely Section 2.3.B in [11], the following construction of a ruled threefold underlying the construction of Cutkosky [5]. We need this for our Example 2.11 and in Section 3.3

Let $S$ be an irreducible projective surface, $A_{1}, A_{2}$ integral Cartier divisors on it. Put

$$
\mathcal{E}=\mathcal{O}_{S}\left(A_{1}\right) \oplus \mathcal{O}_{S}\left(A_{2}\right)
$$

and let

$$
X=\mathbb{P}(\mathcal{E}) \text { and } L=\mathcal{O}_{\mathbb{P}(\mathcal{E})}(1) .
$$

There is a close relation between the the properties of $L=\mathcal{O}_{X}(1)$ and those of $A_{1}$ and $A_{2}$.

Lemma 4.4 With notation as above,

(i) One has $H^{0}\left(X, L^{\otimes k}\right)=\bigoplus_{a_{1}+a_{2}=k} H^{0}\left(S, \mathcal{O}_{S}\left(a_{1} A_{2}+a_{2} A_{2}\right)\right)$.

(ii) $L$ is ample if and only if $A_{1}$ and $A_{2}$ are ample on $S$.

(iii) $L$ is nef if and only if $A_{1}$ and $A_{2}$ are nef on $S$.

(iv) $L$ is big if and only if some $\mathbb{N}$-linear combination of the $A_{i}$ 's is a big divisor on $S$.

(v) For $m \in \mathbb{N}, L^{\otimes m}$ if free if and only if $m A_{1}$ and $m A_{2}$ are both free on $S$.

In connection with the previous example, we will need the following interesting fact about the volume function not established in the literature so far.

Proposition 4.5 (Künneth formula for the volume) Let $X_{1}, X_{2}$ be irreducible projective varieties of dimensions $n_{1}, n_{2}, L_{1}, L_{2}$ line bundles on the respective spaces. Then

$$
\operatorname{vol}_{X_{1} \times X_{2}}\left(\pi_{1}^{*} L_{1} \otimes \pi_{2}^{*} L_{2}\right)=\left(\begin{array}{c}
n_{1}+n_{2} \\
n_{1}
\end{array}\right) \operatorname{vol}_{X_{1}}\left(L_{1}\right) \cdot \operatorname{vol}_{X_{2}}\left(L_{2}\right)
$$

Proof. The statement follows from the Künneth formula for sheaves and the fact that

$$
\operatorname{vol}_{X}(L)=\lim _{m} \frac{h^{0}(X, m L)}{m^{n} / n !}
$$

in general. 


\section{References}

[1] Bădescu, L.: Algebraic surfaces. Universitext, Springer-Verlag, New York, 2001.

[2] Bourbaki, N.: Lie groups and Lie algebras, Chapters 4-6. Elements of Mathematics, Springer-Verlag, Berlin, 2002.

[3] Campana, F., Peternell, Th.: Algebraicity of the ample cone of projective varieties. J. reine angew. Math. 407, 160-166 (1990)

[4] Cossac, F., Dolgachev, I.: Enriques surfaces I. Progress in Mathematics 76., Birkhäuser, Boston, 1989.

[5] Cutkosky, S.D.: Zariski decomposition of divisors on algebraic varieties. Duke Math. Journal 53 (1986), no.1., 149-156.

[6] Cutkosky, S.D., Srinivas, V.: Periodicity of the fixed locus of multiples of a divisor on a surface. Duke Math. J. 72, 641-647 (1993)

[7] Debarre, O.: Higher-dimensional algebraic geometry. Universitext, Springer-Verlag, New York, 2001.

[8] Demazure, M., Pinkham, H., Teissier, B.: Séminaire sur le singularités des surfaces. Lecture Notes in Mathematics 777., Springer-Verlag, Berlin, 1980.

[9] Ein, L. at al.: Asymptotic invariants of base loci. Preprint, arXiv AG 0308116.

[10] Kovács, S.: The cone of curves of a K3 surface. Math. Ann. 300, (1994), no.4., 681-691.

[11] Lazarsfeld, R.: Positivity in Algebraic Geometry, book in preparation

[12] Looijenga, E.: Invariant theory of generalized root systems. Invent. Math. 61 (1980), 1-32.

[13] Nakamaye, M.: Stable base loci of linear series Math. Ann. 318 (2000), 837-847

[14] Nakamaye, M.: Base loci of linear series are numerically determined. Trans. Amer. Math. Soc. 355 (2003), 551-566

[15] Zariski, O.: The theorem of Riemann-Roch for high multiples of an effective divisor on an algebraic surface. Ann. Math. 76, 560-615 (1962)

Thomas Bauer, Fachbereich Mathematik und Informatik, Philipps-Universität Marburg, Hans-Meerwein-Straße, D-35032 Marburg, Germany.

E-mail: tbauer@mathematik. uni-marburg.de

Alex Küronya, Department of Mathematics, University of Michigan, Ann Arbor, MI 48109, USA

E-Mail: akuronya@umich.edu

Tomasz Szemberg, Akademia Pedagogiczna, Instytut Matematyki, ul. Podchorążych 2, PL-30-084 Kraków; Poland and Universität Duisburg-Essen, Fachbereich 6 Mathematik, D-45117 Essen, Germany

E-mail: szemberg@ap.krakow.pl 